\title{
LES RÉGULATIONS NEUROHORMONALES I)E L'INSTINC'T CONSTRUCTEUR DES OUVRIÈRES D'APIS MELLIFICA. COMPLÉMENTS AUX OBSERVATIONS DE RÖSCH ET DE MOSKOVLJÉVIC.
}

PAR

R. DARCHEN.

Station de Recherches apicoles, Bures-sur-Yvette.

Rösch nous a brossé un tableau des occupations des ouvrières d'Apis mellifica au cours de leur brève existence et a établi un parallélisme rigoureux entre leurs travaux et le développement anatomique de leurs différentes glandes : 1'existence de grosses glandes nourricières entraîne automatiquement le comportement de nourrice, celle des glandes cirières celui de constructrice. Cependant, cet auteur présente une expérience ingénieuse par laquelle il est arrivé à séparer les jeunes et les vieilles Abeilles d'une population et à observer le comportement de ces deux catégories d'Insectes : les glandes de bon nombre de vieux sujets s'accroissent de nouveau, dit-il alors, de façon à nourrir les larves et, en revanche, les jeunes sujets partent butiner plus tôt. Rösch conclut donc que de nombreuses régulations existent tout de même dans les sociétés d'A pis mellifica et que 1'on assiste à des régénérations d'organes utiles au maintien de 1'équilibre social. Cependant, ajoute-t-il, après le $26^{\mathrm{e}}$ jour les glandes ne sont plus capables de reprendre un fonctionnement normal.

Cette dernière remarque a été à l'origine de cette étude, car, en plusieurs occasions, il nous avait semblé que des Abeilles bien plus vieilles édifiaient des rayons de cire.

D'autre part, les conclusions des travaux de Moskovljévic ne paraissaient pas s'accorder avec celles de Rösch : les Abeilles du premier, âgées de 75 jours, édifiaient encore quelques bâtisses, et possédaient des glandes très développées. 
Mes expériences ont donc été effectuées sur 6 populations d'Abeilles orphelines : 2 d'entre elles étaient constituées d'Abeilles ayant hiverné, les 4 autres étaient formées à partir du mois de mai avec des Insectes de tous âges. I,es 2 premières devenues orphelines au cours de 1'hiver, étaient composées de quelques centaines d'individus ; les 4 dernières en revanche étaient beaucoup plus populeuses : on pouvait dénombrer environ 5 à I5 ooo sujets. I,es ruchettes, formées de deux cadres construits encadrant un cadre vide, recevaient journellement une ration de sirop de sucre. Enfin, je fixais, de temps en temps, des reines mortes l'année même sur les cellules d'un des rayons construits, en face du cadre vide.

I,es expériences et les résultats peuvent être résumés dans les tableaux suivants.

\section{A. - Utilisation de populations formées au printemps.}

I $^{\text {re }}$ population : 5 ooo Insectes environ.

2-6-I958: formation de la colonie.

I2-6-I958 : début des constructions.

du. I2-6 au 3-7-I958 : constructions journalières.

du 4-7 au 7-7-I958 : aucune construction.

7-7-1958: introduction d'une reine morte entière.

8-7-I958 : nouvelles constructions $(20 \mathrm{~cm}$.).

du 8-7 I958 au I7-7-I958 : constructions journalières $\left(20 \mathrm{~cm}^{2}\right)$. Ellimination de la reine morte à la fin de cette période.

I8-7-I958 : aucune construction. Introduction de la tête de la reine précédente.

I9-7-I958 : constructions nouvelles $\left(20 \mathrm{~cm}^{2}\right)$. Remplacement de la tête par le thorax.

20-7-I958 : constructions ( $\mathrm{I}_{5} \mathrm{~cm}^{2}$ ). Remplacement du thorax par l'abdomen.

2I-7-I958 : constructions ( $\mathrm{I}_{5} \mathrm{~cm}^{2}$ ). Elimination de l'abdomen.

22-7-1958: ancune construction.

23-7-I958 : aucune construction. Introduction de la tête de la reine.

24-7-I958 : aucune construction. Introduction d'une nouvelle reine morte.

du 25-7 au 30-7-I958 : aucune construction.

$2^{\mathrm{e}}$ population : 5000 Insectes.

2-6-r958: formation de la colonie.

9-6-I958 : début des constructions.

du 9-6 au 9-7-I958 : constructions régulières.

I0-7 et II-7-I958 : aucune construction.

I2-7-I958 : aucune construction. Introduction d'une reine morte. 
du I3-7 au I7-7-I958 : aucune construction. Il n'y a.presque plus d'Abeilles.

Introduction d'une reine vivante dans une cagette à la fin de cette période.

du I9-7 au I9-7-I958 : aucune construction.

$3^{\mathrm{e}}$ population : 5 ooo Insectes.

5-6-I958 : formation de la colonie.

II-6-I958 : début des constructions.

du II-6 au I-7-I958 : les Abeilles ne construisent pas régulièrement tous les jours.

du I-7 au 7-7-I958 : aucune construction. Introduction d'une reine morte à la fin de cette période.

du 8-7 au I6-7-I958 : aucune construction.

$4^{\mathrm{e}}$ population : I5 ooo Insectes.

4-7-I958 : formation de la colonie.

6-7-I958 : début des constructions.

du 6-7 au I5-7-I958 : constructions journalières.

du I6-7 au 23-7-r958: aucune construction. A la fin de cette période, introduction de l'abdomen d'une reine ayant déjà été léchée.

24-7-I958 : aucune construction. Introduction d'une reine morte entière.

25-7-I958 : aucune construction. Introduction d'une reine morte supplémentaire.

du 26-7 au 28-7-I958: constructions régulières (I,4, $\left.23 \mathrm{~cm}^{2}\right)$.

\section{B. - Utilisation de populations ayant hiverné.}

$\mathrm{I}^{\text {re }}$ population : orpheline en mars.

4-6-I957 : les Abeilles ne construisent plus depuis bien longtemps.

Introduction d'une reine vierge morte.

5-6-I957 : début des constructions (IO $\mathrm{cm}^{2}$ ).

du 5-6 au ro-6-r957 : constructions journalières.

$2^{\mathrm{e}}$ population : orpheline en février.

Io-6-I958 : nourrissement intense de cette population qui ne construit plus.

du II-6 au I3-6-I958: aucune construction.

I3-6-I958 : introduction d'une reine morte.

I4-6-I958: constructions $\left(20 \mathrm{~cm}^{2}\right)$. Élimination de la reine.

I5-6-I958 : aucune construction. Nouvelle introduction de la même reine. 
I6-6-I958: constructions $\left(30 \mathrm{~cm}^{2}\right)$. Enlèvement de la reine. I7-6-1958 : aucune construction. Dépôt de la tête de la reine. I8-6-I 958: constructions $\left(20 \mathrm{~cm}^{2}\right)$. Je retire la tête. I9-6-1958 : aucune construction. Introduction de la tête. 20-6-1958 : constructions $\left(20 \mathrm{~cm}^{2}\right)$. Élimination de la tête. 2r-6-1958 : aucune construction. Fixation du thorax à l'intérieur de la ruche. 22-6-1958 : constructions $\left(\mathrm{I}_{5} \mathrm{~cm}^{2}\right)$. J'enlève le thorax. 23-6-1958 : aucune construction. Introduction de l'abdomen. 24-6-I958 : constructions $\left(\mathrm{I} \mathrm{cm}^{2}\right)$. Ellimination de l'abdomen. 25-6-I958 : aucune construction. J'introduis à nouveau l'abdomen. 26-6-I958 : aucune construction. Introduction d'une nouvelle reine morte. du 27-6 au 30-6-1958 : aucune construction.

\section{CONCLUSIONS}

$I^{0}$ Les résultats des expériences précédentes nous montrent encore une fois le rôle indéniable de la reine d'A pis mellifica sur le comportement constructeur des ouvrières; $2^{\circ}$ ils nous prouvent en outre que chacune des trois parties du corps de la reine peut posséder une action efficace sur cet instinct; cependant, d'après nos remarques antérieures et les travaux de PaIN, toutes ces parties n'ont pas la même efficience : la tête de la reine provoque des constructions plus grandes que ne le font le thorax et surtout l'abdomen; $3^{0}$ les réactions de la $4^{\mathrm{e}}$ population d'été nous montrent que la substance recueillie sur la reine par les ouvrières doit être en quantité suffisante : devtx reines mortes ont été indispensables pour réactiver l'activité constructrice; $4^{\circ}$ il semble bien aussi que les Abeilles d'hiver possèdent plus de potentialités constructrices que les Abeilles d'été parce que toutes les expériences effectuées avec les premières ont toujours réussi et parce que la réapparition de l'instinct de construction peut se produire chez des Abeilles beaucoup plus âgées en hiver; $5^{\circ}$ contrairement aux opinions émises jusqu'ici, nos expériences indiquent qu'il est possible de réactiver le comportement constructeur chez de très vieilles Abeilles: celles d'été étaient âgées de 60 jours à la fin de l'expérience, celles d'hiver de go jours au début du travail et de ro7 jours à la fin.

Enfin, si l'on se souvient que les travaux de Röscr nous indiquent qu'autour du $25^{\mathrm{e}}$ jour de leur existence les glandes cirières des Abeilles sont presque inexistantes, on est amené à noter plusieurs remarques capitales. Tout d'abord, l'action de la reine entière ou d'une partie de son corps agit sur le système nerveux central des Insectes avant d'entraîner une régénération des glandes cirières étiolées parce que $I^{\circ}$ chez toutes les vieilles populations, les constructions sont reprises immédiatement et qu'elles 
prennent de grandes proportions sans rapport avec la sécrétion de la cire des vieilles glandes cirières, $2^{\circ}$ la cire utilisée en premier lieu, est foncée et donc récupérée en grande partie sur les rayons voisins construits. Bref, le comportement constructeur se développe à la fin de l'existence des Abeilles avant même que les glandes cirières ne soient capables de reprendre une activité régulière : 1'activité constructrice est en avance de phase sur l'activité des glandes cirières.

\section{REFERENCES BIBLIOGRAPHIQUES}

DARCHEN (R.). - La reine d'Apis mellifica, les ouvrières pondeuses et les constructions. Ins. Soc., 4, 321-325, I957.

Les techniquesde construction chez Apis mollifica. Ann. Natur., 12, II3-209, I959.

MoskovlJEVIC (en russe). - Bee World, 20, 83, I939; 21, 39-4I, I940.

PAIN (J.). - En cours de publication.

Rösch (G.). - Untersuchungen über die Arbeitsteilung im Bienenstaat. Z.f. Vergl. Physiol., 2 (6), 57I-63I, I925.

Bautätigkeit und Altder Bienen. $Z$.er $f$. Vergl. Physiol., 6 (2), 264-98, r927. 